\title{
Cyclic nucleotide-gated channels: structural basis of ligand efficacy and allosteric modulation
}

\author{
JUN LI ${ }^{1}$, WILLIAM N. ZAGOTTA ${ }^{2}$ and HENRY A. LESTER ${ }^{1 *}$ \\ ${ }^{1}$ Division of Biology, I56-29, California Institute of Technology, Pasadena, CA 9II25, USA \\ ${ }^{2}$ Department of Physiology and Biophysics, Howard Hughes Medical Institute, University of Washington \\ School of Medicine, Box 35729o, Seattle, WA 98195-729o, USA
}

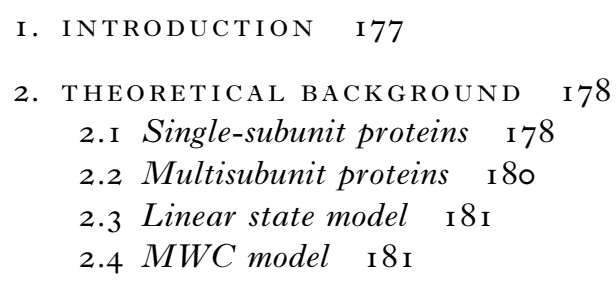

3. LigAND EFFICACY OF CNG CHANNELS: STRUCTURAL BASIS OF $f$ i 83

4. CLASSIFICATION OF FUNCTIONAL MODIFICATIONS I84

5. Allosteric MOdUlations of $L_{0}$ I 86

5. I $\mathrm{Ca}^{2+} /$ calmodulin I 86

5.2 Transition metal ions I 86

5.3 Protons I 87

5.4 Thiol-modifying reagents $\quad \mathrm{I} 87$

5.5 Phosphorylation, etc $\mathrm{I} 88$

6. Modifications of MWC MOdEL i 88

7. CONCLUSION I 89

8. ACKNOWLEDGEMENTS I9O

9. REFERENCES I 90

\section{INTRODUCTION}

Most working proteins, including metabolic enzymes, transcription regulators, and membrane receptors, transporters, and ion channels, share the property of allosteric coupling. The term 'allosteric' means that these proteins mediate indirect interactions between sites that are physically separated on the protein. In the example of ligand-gated ion channels, the binding of a suitable ligand elicits local conformational changes at the binding site, which are coupled to further

* To whom correspondence and reprint requests should be addressed. 
conformational changes in regions distant from the binding site. The physical motions finally arrive at the site of biological activity: the ion-permeating pore. The conformational changes that lead from the ligand binding to the actual opening of the pore comprise 'gating'. In 1956, del Castillo and Katz suggested that the competition between different ligands at nicotinic acetylcholine receptors (nAChRs) could be explained by formation of an intermediate, ligand-bound, yet inactive state of the receptor, which separates the active state of the receptor from the initial binding of the ligand (del Castillo \& Katz, I 957). This 'binding-thengating', two-step model went beyond the then-prevailing drug-receptor model that assumes a single bimolecular binding reaction, and paralleled Stephenson's conceptual dichotomy of 'affinity' and 'efficacy' (Stephenson, I956). In r 965 Monod, Wyman and Changeux presented a simple allosteric model (the MWC model) (Monod et al. i 965) that explained the cooperative binding of oxygen to haemoglobin; it was adopted as an important paradigm for ligand-gated channels soon after its initial formulation (Changeux et al. I 967 ; Karlin, i 967 ; Colquhoun, I 973).

In recent years, molecular cloning and site-directed mutagenesis of ion channels have enabled new insights into the structural basis of the allosteric transitions. Cyclic nucleotide-gated $(\mathrm{CNG})$ channels provide a particularly compelling subject for at least two reasons. (a) Most ligand-gated channels eventually close even in the continued presence of agonist; this phenomenon is called 'desensitization'. The CNG channels, however, display little desensitization, thus eliminating the associated technical and analytical complications. (b) The cyclic nucleotidebinding structures of several cytosolic proteins have been determined by X-ray crystallography, generating some high-resolution hypotheses about CNG channels, which in turn serve to guide site-directed mutagenesis. Thus, despite our ignorance about the structure of CNG channels at any level - except for some basic ideas about transmembrane topography-CNG channels still have great advantages over other channels, and important inferences have been made about their structure, and about structural changes during channel gating. These inferences were derived almost entirely from electrophysiological analysis of wildtype and mutated channels. In this review we focus on studies that reveal structural motifs involved in binding and gating in CNG channels, and the implications of these studies in terms of channel gating mechanisms. From the perspective of the MWC model we propose a phenomenological scheme to classify different functional modifications into a few major types. Finally we review the allosteric modulations of CNG channels by various physiological inputs. Other aspects of CNG channels, such as their molecular cloning, permeation, and physiological function, have been reviewed elsewhere (Finn et al. i 996; Zagotta \& Siegelbaum, r 996).

\section{THEORETICAL BACKGROUND}

\section{I Single-subunit proteins}

We begin by outlining some general principles (see Jackson (r993), Leff ( I995) and Wyman \& Gill (r990) for broader treatments). The simplest allosteric 
receptor possesses one binding site, and can occupy two stable and interconvertible functional states (Fig. I). These two states are usually termed 'inactive' and 'active' for regulated enzymes, G protein-coupled receptors, etc, or 'closed' and 'open' for ion channels. The distribution of the unliganded receptor population between these two states is characterized by a dimensionless equilibrium activation constant, $L_{0}$ (= [active]/[inactive], in the absence of ligand). An allosteric ligand binds to the inactive receptor with equilibrium association constant $K$ (units of $\mathrm{M}^{-1}$ ), and to the active receptor with the different affinity $f K . f$ is thus the ratio between the affinity for the active and inactive states; $f>\mathrm{I}$ for an agonist. Since the free energy difference between two states is the same for all paths connecting them, the agonist-bound receptor must display a higher equilibrium activation constant $\left(f L_{0}\right.$ versus simply $\left.L_{0}\right)$ than the unbound receptor. The linkage is reciprocal: just as the receptor activation stabilizes agonist binding, the agonist binding preferentially stabilizes the active state of the receptor.

The reciprocal interaction between receptor activation and ligand binding strongly resembles the interactions between two ligand binding sites. One classic example for the latter is the mutual interference between the binding of $\mathrm{O}_{2}$ and that of $\mathrm{CO}_{2}$ to haemoglobin - the famous Bohr effect. Just as the binding of $\mathrm{O}_{2}$ destabilizes the binding of $\mathrm{CO}_{2}$ by a factor of $f$, the latter also destabilizes the former, and by the same factor.

Not all ligands that bind to a given site have the same initial binding affinity $(K)$, nor do they have the same potency $(f)$, once bound, in shifting the equilibrium between the functional states available to the receptor. In real cases, a ligand with low initial affinity $K$ may be able to drive the receptors completely into the active state at high enough concentrations; this situation would be characterized by $f L_{0} \gg \mathrm{I}$ in the allosteric model. A high-affinity ligand, on the other hand, may still be a partial agonist, unable to activate the maximum attainable response even at maximum receptor occupancy; this situation would be summarized by a small $f L_{0}$. Thus, the direction and extent of ligand action constitute a continuous spectrum. Based on the values of $f$, one can classify all ligands that reversibly bind to the same site as follows: agonist $(f>\mathrm{I})$, inverse agonist $(f<\mathrm{I})$, and antagonist $(f=\mathrm{I})$. (In the analogous case of interactions between binding sites, one can similarly define positive cooperativity, negative cooperativity (as in the Bohr effect), and independent binding, i.e. the lack of cooperativity between sites.) This classification scheme conforms to the definitions used in the classical receptor theory, where antagonists, for instance, denote the class of ligands that initiate no response themselves, but inhibit the action of agonists or inverse agonists by competing for their binding sites (Stephenson, I 956; Ross, I 996). But compared to the operational definitions used in the classical receptor theory, the definitions based on the allosteric model are more precise, and carry clearer mechanistic implications: an antagonist binds equally well to the active and inactive state, thereby contributing no free energy to alter the spontaneous equilibrium between them; while an inverse agonist binds more tightly to the inactive state, promoting the conversion from active to inactive. For a system with no spontaneous activity, of course, inverse agonists would act as antagonists, in that both activate no 
response but can compete with agonists. Caveats like this call for the rigorous usage of terminology. The term 'antagonist', for example, has been used loosely (I) for all ligands that suppress an agonist response-this category includes inverse agonists, and partial agonists with relatively smaller $f$; or (2) for inhibitors that bind to a different site and exert negative 'heterotropic' effects on the agonist action at the original site - these 'noncompetitive antagonists' are in fact inverse agonists at their own binding site.

\subsection{Multisubunit proteins}

These concepts are straightforward when applied to receptors with a single binding site and a single transition. Allosteric theory is also well suited for describing proteins that contain multiple subunits. The site of biological activity can be confined within each subunit, or it can be located at the interface between subunits. Ion channels typify the latter case: the central aqueous pore is lined by membrane-spanning domains from several identical or homologous subunits. In these multisubunit proteins there may still be allosteric interactions within subunits; but additionally, interactions between subunits strongly influence the conformational changes that govern function as measured experimentally. For instance, an $\mathrm{O}_{2}$-binding site in haemoglobin binds $\mathrm{O}_{2}$ at a higher affinity if one or more neighbouring subunits have already bound; and the activation of a multimeric ligand-gated channel often requires the binding of multiple ligands to their respective sites, usually one on each subunit.

In studying ligand-gated ion channels, many experimenters have measured the fraction of open channels in response to varied concentrations of agonist. In a very common finding, these dose-response relations trace an S-shape rather than a rectangular hyperbola (when the ligand concentration is plotted on a linear scale), yielding $H>\mathrm{I}$ when fit to the Hill equation

$P=\frac{P_{\max }}{\mathrm{I}+\left[E C_{5} \circ / A\right]^{H}}$

where $A$ is the ligand concentration, $P$ is the response, $P_{\max }$ is the maximum response, $E C_{5} \circ$ is the concentration that elicits $50 \%$ of the maximum response, and $H$ is the Hill coefficient. Dose-response data for CNG channels, ever since the pioneering studies (e.g. Fesenko et al. I 985 ; Haynes \& Yau, I 985 ; Zimmerman \& Baylor, I 986), have almost always generated S-shaped curves. The mechanistic origin of an S-shape dose-response is the following: the channel is more likely to open with the presence of multiple bound agonist molecules than with a single bound agonist molecule.

One must distinguish between two quantities that depend on agonist concentration: (I) the fraction of receptors bound with agonist and (2) the fraction of receptors in the active state. At ligand-gated channels - unlike at most soluble proteins - it is rather routine to measure the latter but technically challenging to measure the former. The CNG channels are no exception: even though the channel activation is consistent with $H>\mathrm{I}$, we do not know whether the binding 
curves trace an S-shape rather than a hyperbola. Thus we cannot tell whether there are direct cooperative interactions between the binding sites.

The Hill equation was commonly used for fitting activation curves, because its three parameters, $P_{\max }, E C_{5}$ o, and the Hill coefficient $H$, describe respectively the maximum response to an agonist (relative to a 'maximum attainable response', which can be defined in many different ways), its apparent sensitivity, and the sigmoidicity of the S-shape. A simple linear transformation, the Hill plot, reveals without numerical curve fitting the participation of more than one agonist molecule in channel activation. Its mechanistic basis, however, is the simultaneous binding to all sites, with the partially liganded states virtually unpopulated due to their exceedingly high free energies (as a result of the strong - in principle infinitely strong - cooperative interactions between binding sites). This physical picture is unrealistic: for most proteins some relatively stable intermediate binding states are expected.

\subsection{Linear state model}

The mechanistic limitations of the Hill equation have led researchers to interpret channel activation behaviour in terms of kinetic models in which the intermediate binding states are preserved. One of the most widely used models, based on the traditional 'binding-then-gating' hypotheses, contains a linear series of binding steps leading to a final 'gating' conformational change. Figure $2 \mathrm{~A}$ shows a diagram of states for a channel with two binding sites which are equal in affinity and independent of each other. In linear models like this, an S-shaped activation curve arises naturally since the active state is reached via the fully liganded inactive state. (Of course these curves become more steeply 'sigmoidal' if the binding sites interact so that the binding of one ligand facilitates the binding of the next ligand by directly increasing the affinities of the vacant sites.)

Compared to the model in Fig. I, the gating constant $L$ in the linear model offers a slightly different way to determine whether the ligand is a full or a partial agonist. The normalized maximum response $P_{\max }(=L /(L+\mathrm{I}))$ approaches I when $L \gg \mathrm{I}$, which defines a full agonist. $P_{\max }$ is much less than $\mathrm{I}$ when $L$ is close to or smaller than $\mathrm{I}$, which defines a partial agonist. $L$ also affects the concentration that elicits $50 \%$ of the maximum response $\left(E C_{50}\right)$ and the overall sigmoidicity $(H)$ of the activation curve as quantified by the Hill equation. $K$ and $L$ of a linear model conventionally represent the classical concepts of 'binding' and 'gating': they quantify 'affinity' and 'efficacy' respectively. Because $L$ influences all three Hill parameters, it is incorrect to equate changes in $E C_{5} \circ$ with changes in binding affinity $K$, and it is therefore incorrect to invoke changes in Hill parameters as criteria for distinguishing a given manipulation's specific effects on binding from those on gating.

\subsection{MWC model}

We now take another step in complexity. If the channel can open when only partially liganded, or even completely unliganded, the linear model can be 
expanded into a state matrix with multiple open states, including open states that have no bound agonist. The simplest form, the MWC model, assumes that the binding sites are equivalent, and that upon the concerted transition between the closed and open states these sites remain equivalent, but all have a higher affinity in the open states than in the closed state. The four-site model shown in Fig. 2 B is often adopted for CNG channels because of their tetrameric structure. The horizontal arrows in Fig. $2 \mathrm{~B}$ denote ligand binding, while the vertical arrows denote the transition between the two conformational states: closed and open. The opening of the unliganded channel is usually rather unfavourable $\left(L_{0} \ll \mathrm{I}\right)$, but as the open channels bind agonist molecules more tightly than the closed channels ( $f K$ vs. $K, f>\mathrm{I}$ ), each additional binding enhances the stability of the open conformation by an additional factor of $f$. A channel with all four sites occupied is thus favoured to open by $f^{4} L_{0}$. As the simplest allosteric model for multi-site receptors, the MWC model still follows an S-shaped activation curve, because the channels with greater numbers of bound agonist are more likely to stay in the open states.

Consider a simple case: (a) $L_{0} \ll \mathrm{I}$, so that the constitutive activity is undetectable; and $(b) f \gg \mathrm{I}$, so that the open probability of even the triplyliganded channel $\left(f^{3} L_{0}\right)$ is negligible when compared to that of the fully liganded channel $\left(f^{4} L_{0}\right)$. This case reduces the MWC model to a linear model with four sequential bindings and a final gating step. Thus there is no fundamental difference between the traditional linear model and the MWC model: the distinction is an artificial one, a matter of approximation. The linear model is often adequate for describing actual dose-response data, and it is often preferred for the added advantage of having fewer free parameters. The appeal of the MWC model, on the other hand, lies in its elegance in form, its plausibility in molecular mechanism, its flexibility in allowing spontaneous activities, and its capacity to separate two aspects of gating behaviour, one being the receptor's intrinsic tendency to open $\left(L_{0}\right)$, another being an individual ligand's unique potency in enhancing this tendency $(f)$.

The MWC model also allows the distinction between a ligand's affinity for the inactive state $(K)$ and that for the active state $(f K) . \Delta G_{\text {coupling }}=-R T \ln f$ represents the difference between the free energy changes involved in the high and low affinity bindings; this is the same amount that each binding reaction contributes to overcoming the intrinsic gating barrier $\Delta G_{\text {intrinsic }}=-R T \ln L_{0}$ ( $>$ o when $L_{0}<\mathrm{I}$ ). It is because of the difference in binding properties of different protein conformations that binding can in turn affect the population of these conformations.

If the symmetry between binding sites is not preserved because of (I) unequal affinities of individual binding sites or (2) direct interactions between them, the expanded MWC model has to include individually-defined initial binding constants. Furthermore if there is more than one conformation for the open channel, or if the conformational changes in different subunits are not concerted, a generalized allosteric model with more than two functional states is to be employed (Hammes \& Wu, r 97 I). The MWC model is just one special case (the concerted-symmetry model) of this general model. Another commonly considered 
special case is the sequential interaction model proposed by Koshland, Nemethy and Filmer, in which the binding of each ligand 'induces' a new conformational state of the receptor, with distinct binding and functional properties (Koshland et al. r 966). These models, however, lie outside the intended scope of this review. Interested readers may refer to the original literature, or to a monograph on this subject by Wyman and Gill (Wyman \& Gill, I990).

\section{LIGAND EFFICACY OF CNG CHANNELS: STRUCTURAL BASIS OF $f$}

A recent study of nucleotide-gated channels best illustrates the concept of $f$. For the CNG channel cloned from bovine rod photoreceptors, cGMP is a full agonist; but cAMP is a partial agonist, activating about $\mathrm{I} \%$ of the maximum current. What is the structural basis for the vastly different efficacies of these two ligands? Varnum and co-workers (Varnum et al. I 995) discovered that residue D604 in the $\mathrm{C} \alpha$-helix of the putative cyclic nucleotide-binding site (Fig. 3 ) accounts for almost all the difference. Neutralizing this residue in mutant D604M reverses the rank order of agonist efficacy - cGMP becomes a partial agonist, and cAMP, while still a partial agonist, now activates a greater amount of current than cGMP. The result is explained in a structural model in which D604 makes two hydrogen bonds with $\mathrm{N}_{1}$ and $\mathrm{N}_{2}$ of the guanine ring of cGMP, while it generates a repulsive interaction with the non-bonding electrons in the $s p^{2}$ orbital at $\mathrm{N}_{\mathrm{I}}$ in cAMP (Fig. 4). Using the linear model shown in Fig. $2 \mathrm{~A}$, it was shown that the $L$ values, determined by the maximal responses $\left[P_{\max }=L /(L+\mathrm{I})\right]$ normalized against the maximal attainable current measured under saturating conditions, by themselves fit the observed changes in dose-response, including the shift in sensitivity. Thus the initial binding affinity $K$ is unlikely to be greatly affected by the various mutations at residue 604; the primary effect of such mutants is to alter the gating constant $L$, distinctively for cAMP and cGMP. The structural model further postulates that after the ligand has docked to an 'initial binding site', D604 pulls on the Chelix, so that the latter would rotate with respect to other components of the cyclic nucleotide-binding site such as the $\beta$-roll (Fig. 4). This rotation is coupled to the opening of the channel, via 'linkage' structures that are at present unknown. Chelix rotation with respect to other domains of the protein complex has been implicated in the activation of $E$. coli catabolite activating protein (CAP) as well (Weber \& Steitz, I987; Heyduk et al. r 992).

Interpreted according to the classic MWC model (Fig. 2 B), the effect of D604 mutants is to modify a ligand-receptor interaction that occurs subsequent to the initial binding and is congruent with the gating transition - exactly the coupling process embodied in $f$. The differences of $L$ (in Varnum et al.'s linear model) among ligands, and among channel mutants, arise from the differences in $f$. These differences can be easily incorporated into the MWC model to deal with situations that involve mixtures of ligands or channels with mixed subunits (e.g. Varnum \& Zagotta, i 996).

The $f$ values of ligands constitute their distinct allosteric potency; $f$ is therefore perhaps the most meaningful (though comparative) definition of ligand efficacy. 
Previously we discussed that it is almost impossible to separate 'affinity' and 'efficacy' by analysing dose-responses; here it can be appreciated that this difficulty arises because the apparent affinity $E_{5}$, being a weighted average of $K$ and $f K$, depends inherently on ligand efficacy $f$.

\section{CLASSIFICATION OF FUNCTIONAL MODIFICATIONS}

Environmental inputs constantly impinge on a given receptor-ligand complex. The literature on ligand-gated channels shows many examples of modification of function by physical conditions such as temperature, $\mathrm{pH}$, membrane composition and membrane tension; by point mutations or chimeric constructions; by covalent modifications such as phosphorylation; by interactions with other proteins; and by pharmacological or chemical treatments. The MWC model provides the conceptual framework for categorizing these modifications into three major types (for a similar scheme see (Galzi et al. r 996)): those primarily affecting $K$, those affecting $f$ and those affecting $L_{0}$. In discussions below we focus on examples from CNG channels, for which some of the modifications mentioned above have already been examined.

A $\boldsymbol{K}$ type modification would take place at the initial binding site. It does not change a channel's intrinsic gating tendency $\left(L_{0}\right)$, nor is it involved in the liganddependent component of gating $(f)$. Altenhofen et al. found that $\mathrm{T}_{5} 60$ in the cyclic nucleotide-binding domain of the bovine rod CNG channel may form a hydrogen bond with the $\mathrm{N}_{2}$ group of cGMP (Altenhofen et al. I99I). Mutant $\mathrm{T}_{5} 60 \mathrm{~A}$ reduces the $E C_{5}$ o of cGMP by 30 -fold, but does not affect the $E C_{5}$ o of cAMP, for which this bond cannot be formed. Similarly, Tibbs et al. found that $\mathrm{R}_{559} \mathrm{~A}$ reduces cGMP sensitivity by 25 -fold (Tibbs et al. I996). In structural models based on the sequence similarity between the binding sites on CNG channels and E. coli $\mathrm{CAP}$ - the structure of which is known - both $\mathrm{T}_{5} 60$ and $\mathrm{R}_{559}$ interact directly with the cyclic nucleotides (Kumar \& Weber, I992; Scott et al. 1996) (Fig. 4). However, $\mathrm{T}_{5} 60 \mathrm{~A}$ is not purely a $K$ mutant: the quantitative analysis by Varnum et al. (Varnum et al. I 995) shows that $\mathrm{T}_{5} 60 \mathrm{~A}$ has an additional effect on $L$. It is worth noting that the estimation of $L$ is not very sensitive to the specific hypotheses regarding binding steps or the partially liganded openings: the open probability measured at saturating concentrations uniquely defines $L$, while the best-fitting values of $K$ depend on the number of binding steps assumed, and on other assumptions about equivalency among binding sites.

Changes that affect $f$ would also occur at the binding site, like changes that affect $K$. But the $f$ type changes, unlike the $K$ type, can affect the ligands' differential abilities to stimulate the response. Thus, changes in $f$ are often reflected by differences in $P_{\max }$, both among different ligands for the same channel and among different channel mutants activated by the same ligand. In the examples discussed earlier, D604 of the bovine rod channel is strongly involved in determining the differential efficacies of cGMP and cAMP (Varnum et al. I 995).

Different CNG channels apparently interact with distinct structural moieties of the ligand during the gating transitions (Kramer \& Tibbs, I 996). The bovine rod 


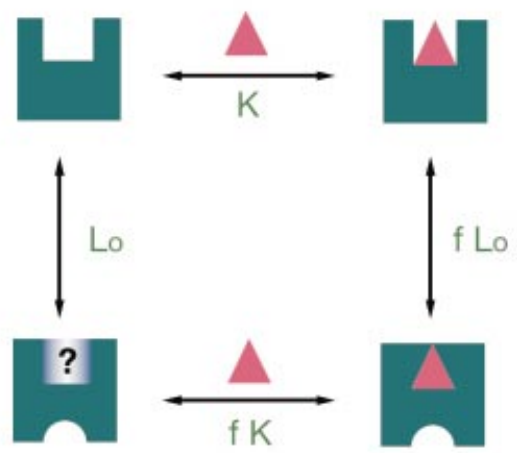

Fig. 1. A one-site, two-state allosteric receptor. The four states are, clockwise from top-left: unliganded/inactive, liganded/inactive, liganded/active, unliganded/active. $\mathrm{K}$ is the equilibrium association constant of the inactive states (unit: $\left.\mathrm{M}^{-1}\right)\left(=1 / \mathrm{K}_{\mathrm{d}}, \mathrm{K}_{\mathrm{d}}\right.$ is the more frequently used equilibrium dissociation constant). $\mathrm{L}_{0}$ is the equilibrium ratio of active to inactive states in the absence of the ligand. $\mathrm{f}$ is the factor by which receptor activation stabilizes binding, or reciprocally, the factor by which ligand binding stabilizes activation. Upon activation, the binding site acquires a new configuration that complements the structure of the agonist molecule. The "?" in the unliganded/active receptor indicates that we do not know whether the spontaneous activation of the unliganded receptor is always accompanied by the unoccupied binding site adopting the precise ligand-bound configuration.

A.

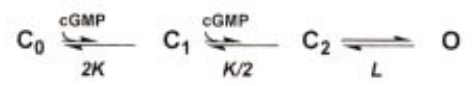

B.

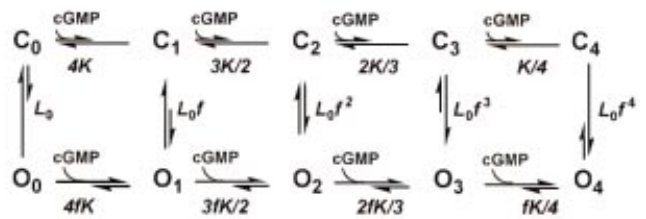

Fig. 2. A. The linear binding model for a cGMP-gated channel with two equivalent, independent binding sites. $\mathrm{C}_{0}-\mathrm{C}_{2}$ are the closed states; $\mathrm{O}$ is the sole open state. The channel can open only when doubly-liganded. K: equilibrium association constant (unit: $\mathrm{M}^{-1}$ ) of the individual binding site; $\mathrm{L}$ : equilibrium "gating" constant between $\mathrm{C}_{2}$ and $\mathrm{O}$. Weighing factors of 2 and $1 / 2$ multiply $\mathrm{K}$ to reflect an emergent statistical feature of two-site receptors: there are two sites available for binding for the first ligand, which can dissociate in only one way; while the second ligand can be added in only one way, but can be removed from either one of the two sites.

B. The Monod-Wyman-Changeux (MWC) model for a cGMP-gated channel with four equivalent, independent binding sites. $\mathrm{C}_{0}-\mathrm{C}_{4}$ are the closed states; $\mathrm{O}_{0}-\mathrm{O}_{4}$ are the corresponding open states. K: microscopic equilibrium associating constant of the closed states; $\mathrm{L}_{0}$, equilibrium constant between closed and open states in the absence of ligand; $f$ : the multiplicative factor with which each binding displaces the closed-open equilibrium. Statistical factors of $4,3 / 2,2 / 3$, and $1 / 4$ multiply the association rate constant $\mathrm{K}$ of individual binding site. Note that $\mathrm{L}_{0}$ and $\mathrm{f}$ are reciprocals of the $\mathrm{L}$ and $\mathrm{c}$ in the original MWC model (Monod et al. 1965). 


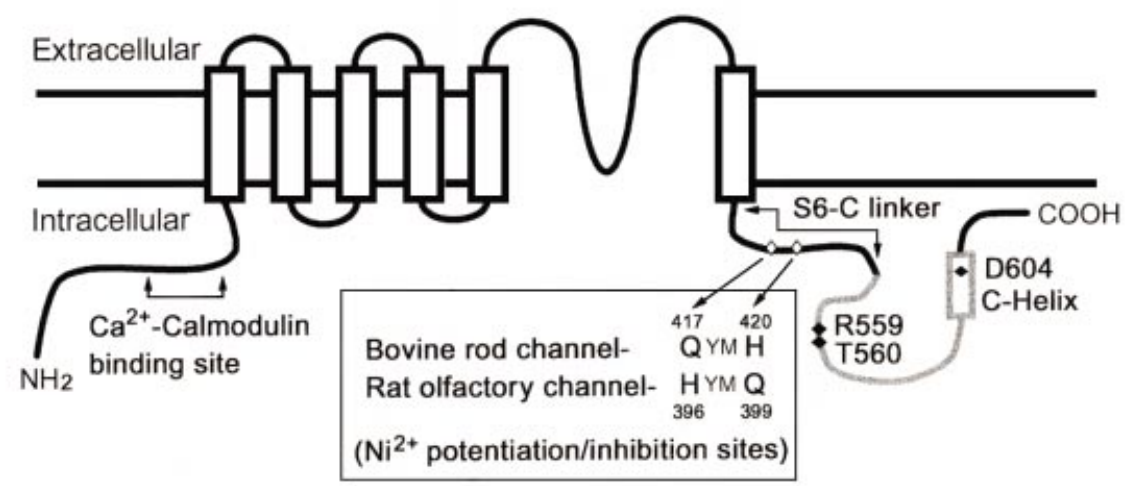

Fig. 3. The generalized structure of a typical $\alpha$ subunit of CNG channels, showing the putative transmembrane topology, and locations of the residues/domains discussed in this review. The amino acids are numbered according to the bovine rod channel. The histidine residues involved in $\mathrm{Ni}^{2+}$ potentiation (bovine rod channel) and inhibition (rat olfactory channel) are highlighted in the box. The cyclic nucleotide binding domain, including the $\mathrm{C} \alpha$-helix, is shaded. Lengths of different protein segments are not drawn to scale.

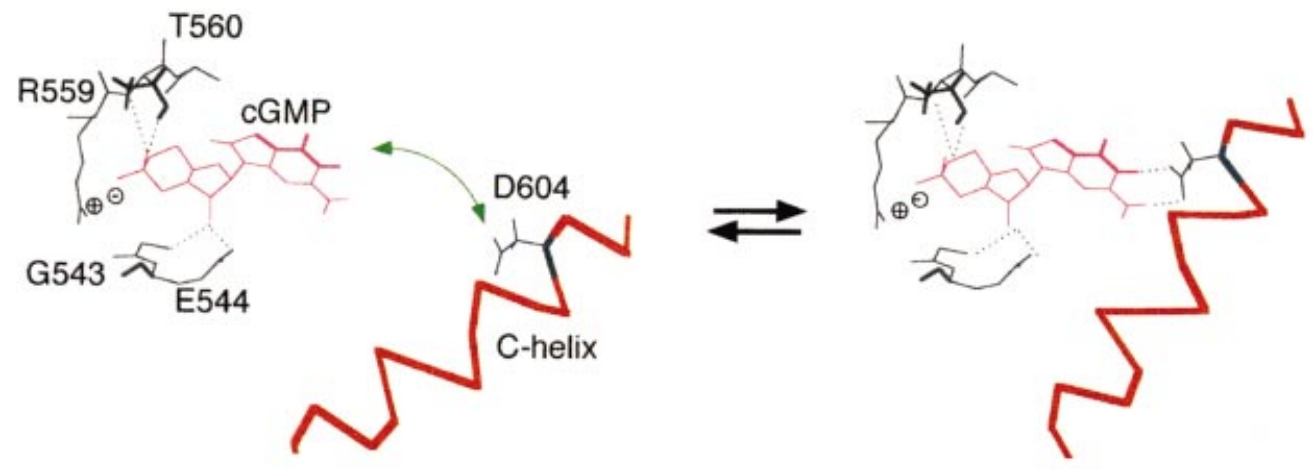

Fig. 4. Proposed conformational changes at the binding site during CNG channel gating. Shown is a structural model of the cyclic nucleotide-binding site of the bovine photoreceptor channel. The model is generated based on the sequence similarity between CNG channels and the E. coli CAP, as described in detail before (Varnum et al. 1995). Upon the "docking" of cGMP in the binding site of a closed channel, the formation of hydrogen bonds between D604 and the N1 and N2 hydrogens of cGMP is accompanied by the rotation of the $\mathrm{C}-\alpha$ helix, which is "hinged" at the junction between the $\mathrm{C}-$ and $\mathrm{B}-\alpha$ helix (the latter not shown). C helix rotation drives further unknown conformational changes that lead eventually to channel opening. The amino acid residues are numbered according to the bovine rod channel. Only the anti conformation of the cGMP molecule is shown. Other researchers have proposed that cGMP binds in the syn conformation (e.g. (Altenhofen et al. 1991; Kumar \& Weber, 1992)). 


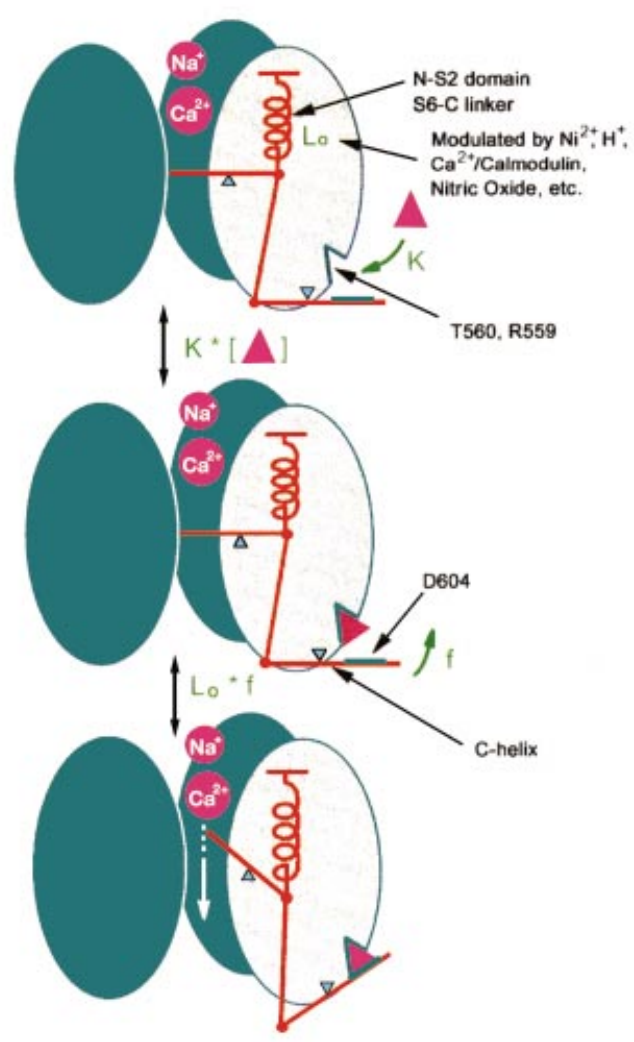

Fig. 5. A highly idealized cartoon summarizing key features of CNG channel function. Shown are 3 subunits of a tetrameric CNG channel, with one subunit sliced open, exposing the binding and gating structures. A cyclic nucleotide molecule - depicted as the pink triangle - binds to its initial binding site with association constant $\mathrm{K}$. This initial binding site, depicted as the triangular indent on the subunit, includes T560 and R559 (Altenhofen et al. 1991; Tibbs et al. 1996): of course some other unidentified residues may also be involved. The binding induces the rotation of the C-helix, which in turn is stabilized by the interaction between the ligand molecule and residues on the C-helix including D604 (Varnum et al. 1995). The C-helix contacts the "docked" ligand molecule on its third side, rendering the binding site more complementary to the shape of the ligand. The rotation of the C-helix is coupled, via a gating pathway (the red connected rods), to the opening of the channel, shown very figuratively as the swinging open of the "gate" at the intersubunit passage through which $\mathrm{Na}^{+}$and $\mathrm{Ca}^{2+}$ ions flow. The spontaneous activity is influenced by the N-S2 domain and the S6-C linker (Goulding et al. 1994; Gordon \& Zagotta, 1995b). The multiple determinants of the channel's intrinsic gating activity $\left(\mathrm{L}_{0}\right)$ are categorically represented by the spring. The actions of $\mathrm{Ca}^{2+} /$ calmodulin, $\mathrm{Ni}^{2+}$, proton, nitric oxide, etc (see text), each probably takes place at a different site on the spring. Among these factors, nitric oxide (Broillet \& Firestein, 1996a) is special in that it activates by itself. The identified amino acid residues and channel domains responsible for determining $\mathrm{L}_{0}, \mathrm{~K}$ and $\mathrm{f}$ are designated by arrows. We do not know whether the spontaneous opening (not shown) is accompanied by the movements of binding structures such as the C-helix. This simple cartoon does not reflect intersubunit interactions, nor the fact that with more agonist molecules bound, the channel is more likely to open. 
channel interacts with the purine moiety, and recognises cGMP (or one of its analogs) as a good agonist, and cAMP (or one of its analogs) as a poor agonist (or possibly an antagonist). In contrast, the catfish olfactory channel is indifferent to the identity of the purine but identifies the ribose-phosphate group as the major determinant of gating, so that the $\mathrm{Rp}$-isomers of the thio-derivatives are partial agonists, whereas the Sp-isomers are full agonists-cAMP or cGMP alike (Kramer \& Tibbs, I 996). Such stereoselectivity was also reported for the cAMP(Botelho et al. I 988; Dostmann \& Taylor, I99I), or cGMP-dependent protein kinases (Butt et al. I990) and E. coli CAP (Scholübbers et al. I984).

Unlike the $K$ and $f$ types, an $\boldsymbol{L}_{0}$ type effect usually originates in domains other than, and often distant from, the immediate binding site. The actual $L_{0}$ values of CNG channels are usually small, resulting in barely discernible levels of spontaneous activities. In divalent-free solutions, the CNG channels of the cone photoreceptors of the striped bass have on average $4.5 \%$ basal opening probability (Picones \& Korenbrot, I 995). The cloned catfish olfactory channel shows a basal activity of about $2 \times \mathrm{IO}^{-3}$, whereas the cloned bovine rod channel shows about $\mathrm{IO}^{-5}$ (Tibbs et al. I 996). This latter value is unaffected by the $\mathrm{R}_{559} \mathrm{~A}$ mutation, which lowers the sensitivity to cGMP by 25 -fold (Tibbs et al. i 996) - this rules out the trivial possibility that the apparent unliganded openings are caused by contaminating agonists. Consistent with this result, the spontaneous activation at the striped bass cone photoreceptors was unaffected by treatment with activated phosphodiesterases (Picones \& Korenbrot, I995). As noted before, the MWC model qualitatively anticipates the detection of spontaneous activity. But in one encouraging result, the observed spontaneous open probability actually agrees quantitatively with the $L_{0}$ value derived from the steady-state dose-response data as interpreted by the MWC model (Tibbs et al. I 996). It is expected, but not yet proven, that the various modulations of $L_{0}$ reviewed in the next section are accompanied by appropriate changes in the spontaneous activities.

The difference in $L_{0}$ between catfish olfactory channel and bovine rod channel is ascribed, by examining chimeric constructs, to the $\mathrm{N}$-terminal region together with the first two transmembrane segments (N-S2) (Goulding et al. 1994). Another chimeric study using the bovine rod and rat olfactory channels (Gordon $\&$ Zagotta, r $995 b$ ) concluded that the gating is governed by regions 'distributed' along the primary sequence, including, among others, the N-S2 domain, and the region between the sixth transmembrane segment and the cyclic nucleotide binding domain (S6-C linker) (Fig. 3).

Many mutations or modifications alter the observed maximum response, implying a gating effect. How can one, in general, distinguish $L_{0}$ types from $f$ types? If a mutation affects the actions of different ligands to the same extent, it qualifies as an $L_{0}$ type. For example, in the chimera experiments mentioned above, the N-S2 domain of the olfactory channel supports a higher $P_{\max }$, for both cAMP and cGMP, than does the corresponding domain of the rod channel. On the other hand, if the mutation affects different ligands differently, it must be involved both in the specific recognition of the ligand and in the induced gating transition - an $f$ type. In real experiments, however, the distinction is not always straightforward. 
The effects of $\mathrm{pH}$ on the bovine rod channels, for instance, are carried both by an $L_{0}$ component and by an $f$ component (details below).

\section{ALLOSTERIC MODULATIONS OF $L_{0}$}

Perhaps owing to the fact that there are usually far more channel domains involved in gating than in initial binding, the $L_{0}$ phenotype accounts for the majority of a CNG channel's physiological modulation. The following examples of $L_{0^{-}}$ modulation are confined to $\mathrm{CNG}$ channels. Readers interested in $\mathrm{nAChR}$ or other ligand-gated channels may refer to other reviews (e.g. Galzi \& Changeux, I 994; Changeux, I 995; Smith \& Olsen, I995).

\section{I $\mathrm{Ca}^{2+} /$ calmodulin}

$\mathrm{Ca}^{2+} /$ calmodulin is a well documented feedback-modulator of native or expressed CNG channels (Hsu \& Molday, I993; Chen \& Yau, I 994). The apparent agonist sensitivity of the native rod channels of leopard frog, for example, is reduced by calmodulin and by an endogenous calmodulin-like cytoplasmic protein (Gordon et $a l$. I 995 b). The decrease in cyclic nucleotide sensitivity is, at least for the rat olfactory channel, due primarily to a gating effect, via the stabilization of the closed state by the direct binding of calmodulin (Liu et al. I 994). The calmodulin binding site has been identified on the $\mathrm{N}$-terminal region of the $\alpha$ subunit of the rat olfactory channel (Liu et al. I 994) (Fig. 3) and on the C-terminal region of the $\beta$ subunit of the bovine rod channel (Molday, I996). The recognition of the Nterminal region of the olfactory channel as the calmodulin binding site concurs with the finding that the amino terminal is responsible for a CNG channel's intrinsic tendency to open $\left(L_{0}\right)$ (Gordon \& Zagotta, I 995 b; Goulding et al. I 994). The physiological roles of this modulation have been reviewed recently (Molday, I 996).

\subsection{Transition metal ions}

Cytoplasmic transition metal ions, such as $\mathrm{Ni}^{2+}, \mathrm{Cd}^{2+}, \mathrm{Zn}^{2+}, \mathrm{Co}^{2+}$ and $\mathrm{Mn}^{2+}$, also affect CNG channels (Ildefonse \& Bennett, I99I; Karpen et al. I993). $\mathrm{Ni}^{2+}$ potentiates the rod channel (Gordon \& Zagotta, I $995 a$ ), and inhibits the olfactory channel (Gordon \& Zagotta, I 995 $b$ ). The action of $\mathrm{Ni}^{2+}$ appears to be entirely on gating; this conclusion is drawn from the finding that changing $L$ (in the linear model), to the same extent for cAMP and cGMP, is sufficient to account for most of the observed dose-response changes (Gordon \& Zagotta, r995 $a, b$ ). The potentiation is due to a stabilizing effect on the open states, whereas the inhibition occurs via stabilization of the closed states. The potentiating site on the bovine rod channel is $\mathrm{H}_{42} \mathrm{O}$; the inhibiting site on the olfactory channel is $\mathrm{H}_{39} 6$ (Fig. 3). If one mutates the potentiating histidine from the rod channel $\left(\operatorname{rod} \mathrm{H}_{42} \mathrm{OQ}\right)$ or the inhibiting histidine from the olfactory channel (olf. H396Q), the mutated channels are no longer affected by $\mathrm{Ni}^{2+}$. The two histidine residues are located in the $\mathrm{S} 6-$ 
$\mathrm{C}$ linker, suggesting that this region participates in the conformational changes leading to channel opening (Gordon \& Zagotta, I 995 b). It is not known how many $\mathrm{Ni}^{2+}$ ions are needed for the effect. It is known, however, that each $\mathrm{Ni}^{2+}$ binding is coordinated by at least two histidine residues, one each from two adjacent, but not diagonally opposite, subunits (Gordon \& Zagotta, I 995c).

\subsection{Protons}

Intracellular protons potentiate the expressed bovine rod $\mathrm{CNG}$ channel $\alpha$ subunit with two components (Gordon et al. I996). The first component is cAMPspecific, and is due to the protonation, hence neutralization, of D604, which normally would repel the non-bonding electrons of $\mathrm{N}_{\mathrm{I}}$ in cAMP. Thus protons increase $f$ for cAMP. The second component is independent of ligand. This $L_{0}$ effect is attributed to proton binding to $\mathrm{H}_{4} 68$. The $\operatorname{rod} \beta$ subunit has neither D604 nor $\mathrm{H}_{4} 68$. Both the expressed $\alpha / \beta$ heteromultimer and the native channel are less potentiated than the expressed $\alpha$ homomultimers under comparable conditions (Sanfilippo \& Menini, I 993; Gordon et al. I 996), consistent with the present idea that the native rod channels are $\alpha / \beta$ heteromultimers (Chen et al. r 994; Körschen et al. I 995). The slope of the $\mathrm{pH}$-titration curve indicates that at least two protons are required for the potentiation. It is not clear where the two binding sites are physically located - a presumably tetrameric channel contains four D604s and four $\mathrm{H}_{4} 68 \mathrm{~s}$. It is also not known whether proton potentiation has any physiological function.

\subsection{Thiol-modifying reagents}

Nitric oxide (NO), and several other thiol-modifying reagents, activate the native salamander olfactory channels in the absence of the cyclic nucleotide, possibly via the modification of cysteine residues (Broillet \& Firestein, r 996a). Similarly NO also activates the rat olfactory $\beta$ subunit expressed alone (Broillet $\&$ Firestein, I $996 b$ ), which cannot be activated by cAMP or cGMP (Bradley et al. I 994; Liman \& Buck, I994). These two classes of channel activators, cyclic nucleotides and thiol-modifying reagents, are each sufficient for activating the native salamander olfactory channels. Rp-cAMP, a weak agonist at the cyclic-nucleotide binding site, strongly antagonizes the cAMP activation - presumably by competition - but does not affect the activation by SNC, an NO-donor. On the other hand, DTT, a thiol-reducing agent that suppresses the NO effect, does not affect the activation by cAMP. Sulphydryl reagents such as NO stand out among known modulatory factors of $\mathrm{CNG}$ channels by their ability to activate the channel on their own. It would be interesting to examine more closely the interactions between the gating pathways activated by cyclic nucleotides and by thiol-modifying reagents. The site modified by these reagents has not yet been identified. According to one hypothesis, a cysteine residue in the S6-C linker may be reversibly oxidized. It is interesting to see that the native bovine rod channel was affected by the sulphydryl reagents, but not by NO donors (Serre et al. i 995 ). 


\subsection{Phosphorylation, etc}

Phosphorylations (Gordon et al. I992) and DAG (Gordon et al. I995a) also appear to modulate the native rod channel activity. The sites of action are still not known, although the phosphorylation effect seems to be mediated by two sites, with opposite effects: the channel rundown upon patch excision is mimicked by phosphatase I but reversed by phosphatase $2 \mathrm{~A}$. The phosphorylation and modulation by lipid both act, at least in part, on gating, since in addition to the shifts in apparent sensitivity to cyclic nucleotides the maximum responses are also altered.

\section{MODIFICATIONS OF MWC MODEL}

In applications of the classic MWC model it has been assumed that the open states (Oo- $\mathrm{O}_{4}$ in Fig. $2 \mathrm{~B}$ ) are functionally equivalent except for the number of bound agonist molecules. Applied to $\mathrm{CNG}$ channels, the equivalent assumptions are that ( $\mathrm{s}$ ) all the subunits have the same initial binding affinity ( $K$ when the channel is closed, $f K$ when open) - the hypothesis of equivalent and independent binding, and (2) the open states have the same channel conductance. Yet the exemplar allosteric protein, haemoglobin, is certainly a heterotetramer; and native CNG channels are probably heterotetramers as well (Chen et al. I993; Bradley et al. I 994; Liman \& Buck, I 994). Experimental findings do indeed show that these two assumptions are too simple. In a photoaffinity-ligand study Karpen and Brown (Karpen \& Brown, I996) revealed a surprising lack of functional uniformity among the expressed homomeric channels, and the possible presence of at least two types of binding sites that differ in apparent affinity for cGMP by a factor of $\sim 25$.

In contrast to the hypothesis of equal conductance, several investigators have reported multiple levels of single-channel current. Taylor and Baylor (Taylor \& Baylor, I 995) found that the salamander rod outer segment CNG channels display a subconductance that appears less frequently at high concentrations of cGMP, suggesting that this sub-conductance state represents a partially liganded channel. Ildefonse and Bennett (Ildefonse \& Bennett, r 99I) discovered at least four levels of single-channel conductance in recordings from the bovine rod channels reconstituted into planar lipid bilayers. Perhaps each conductance corresponds to one of the four liganded open states in the original MWC model. Consistent with this hypothesis, the smaller conductances, which might represent partially liganded states, decrease in occurrence at higher concentrations of agonist; at the same time the channel openings become dominated by the largest conductance, presumably fully liganded.

The third possible modification of the MWC model is to include at least one additional closed state to represent the long (tens to hundreds of ms) closed periods observed in the presence of saturating agonist concentrations (Matthews \& Watanabe, I 988; Taylor \& Baylor, I 995). For such a condition the MWC model would predict a simple equilibrium between the fully liganded-closed and the 
fully liganded-open states, manifested by a single exponential component in the duration histogram for either the closed or the open state. Perhaps CNG channels are subject to the scourge of desensitisation after all.

Thus, we anticipate that some modifications to the MWC model will be required to understand all the properties. Macroscopic measurements probably lack the precision to constrain much more than three parameters (e.g. $K, f$ and $L_{0}$ in the MWC model). In this regard single-channel measurements provide a much richer set of data, representing the only present method for monitoring the realtime, single-molecule behaviour of allosteric proteins. Single-channel recordings ( I) detect multiple conductances (and sometimes multiple discrete components in the histograms of the open or closed times) that may reveal intermediate binding states, (2) allow direct measurements of the open probability, and (3) can be used to extract forward and reverse kinetic rates for many allosteric transitions. The single-channel data reported thus far for CNG channels show conductances in the same amplitude range, and kinetics in the same time range, as, for instance, neurotransmitter-gated channels such as nicotinic acetylcholine receptors. We may thus expect kinetic data roughly equal in resolution to those obtained for muscle type nicotinic receptors, where the state-of-the-art models contain as many as eight individual rate constants that govern transitions among four closed and one open states (Zhang et al. I 995). Someday, with improved resolution, we may see CNG channel behaviours that deviate from the basic assumptions of the classic MWC model, such as the symmetry among subunits. But even then, the three parameters in the MWC model, $K, L_{0}$ and $f$, as simple concepts representing initial binding, spontaneous activation and their 'heterotropic linkage', will retain their intuitive meanings, and probably their places in our vocabulary.

\section{CONCLUSION}

The concept of allosteric coupling between binding and gating provides plausible interpretations for most of the recent studies on gating behaviours of CNG channels (Fig. 5). From this common viewpoint we have proposed a system to categorize and quantify various functional modifications. Additionally, our specific model lays out a starting point, from which more elaborate theoretical frameworks may be developed to accommodate additional functional properties.

It is important to emphasize, however, that the MWC model, or any other allosteric hypotheses, despite their widespread success, are still provisional kinetic schemes. Their role is to summarize salient features of experimental results, and to explain these features at a defined level of detail. Sometimes one may find simplifying assumptions helpful in highlighting basic principles, but at other times the goal may be to employ generalized models to capture interesting and complex functional features. The choice is to be made according to the data at hand, and the degree of refinement one seeks - in this regard the MWC model possesses a level of simplification compatible with the knowledge presently available for CNG channels.

The values of $L_{0}$ and $f$ are functions of the entire biological system; therefore 
a proper taxonomy of ligands in terms of agonists $(f>\mathrm{I})$, inverse agonists $(f<$ I), and antagonists $(f=\mathrm{I})$, etc, cannot be unconditionally established. Scientists investigating sensory transduction and adaptation are now faced with formerly unappreciated complexities: even at the level of individual CNG channel proteins, multiple signals interact via the binding-coupled conformational equilibria (Fig. 5). Such complexities also present opportunities: the numerous modes of channel modulation expand our repertoire of tools for exploring, in relative isolation, individual functional states and the elementary steps connecting them. We expect that the allosteric paradigms will continue to guide new discoveries, and that the cyclic nucleotide-gated channels will join the list of exemplar allosteric proteins by contributing greatly to our understanding of allosteric mechanisms as a basic and universal means of biological activation and regulation.

\section{ACKNOWLEDGEMENTS}

The authors thank members of the Zagotta laboratory for stimulating discussions. Sela Mager, Mark Nowak, Yinong Zhang and Jing Liu read the manuscript critically. The preparation of this review is supported by research grants from the National Institute of Health: EY-ı0329, MH-49i76, GM-29836, and by predoctoral training grant GM-0850 I. W. N.Z. is an Investigator of the Howard Hughes Medical Institute.

\section{REFERENCES}

Altenhofen, W., Ludwig, J., Eismann, E., Kraus, W., Bönigk, W. \& Kaupp, U. B. (I99I). Control of ligand specificity in cyclic nucleotide-gated channels from rod photoreceptors and olfactory epithelium. Proc. Natl. Acad. Sci. USA 88, 9868-9872.

Botelho, L., Rothermael, J., Coombs, R. \& Jastorff, B. (i988). cAMP analog antagonists of cAMP action. Meth. Enzym. I59, I 59-172.

Bradley, J., Li, J., Davidson, N., Lester, H. A. \& Zinn, K. (i994). Heteromeric olfactory cyclic nucleotide-gated channels: a subunit that confers increased sensitivity to cAMP. Proc. Natl. Acad. Sci. USA 91, 8890-8894.

Broillet, M.-C. \& Firestein, S. (ig96a). Direct activation of the olfactory cyclic nucleotide-gated channel through modification of sulfhydryl groups by NO compounds. Neuron 16, 377-385.

Broillet, M.-C. \& Firestein, S. ( I $996 b$ ). Homomeric cyclic nucleotide-gated channels formed by cloned rat olfactory subunit 2 are gated by nitric oxide. Abstracts of Society for Neuroscience 22, $65 \mathrm{I}$.

Butt, E., Bemmelen, M. V., Fischer, L., Walter, U. \& Jastorff, B. (i 990). Inhibition of cGMP-dependent protein kinase by $\left(\mathrm{R}_{\mathrm{p}}\right)$-guanosine $3^{\prime}, 5^{\prime}$-monophosphorothioates. FEBS Lett. 263, 47-50.

Changeux, J.-P. (i 995). The acetylcholine receptor: a model for allosteric membrane proteins. Biochem. Soc. Trans. 23, I 95-205.

Changeux, J.-P., Thiéry, J., Tung, Y. \& Kittel, C. (i967). On the cooperativity of biological membranes. Proc. Natl. Acad. Sci. USA 57, 335-34 I.

Chen, T.-Y., Illing, M., Molday, L., Hsu, Y.-T., Yau, K.-W. \& Molday, R. (i 994). Subunit 2 (or $\beta$ ) of retinal rod cGMP-gated cation channel is a component of the $240-$ 
$\mathrm{kDa}$ channel-associated protein and mediates $\mathrm{Ca}^{2+}$-calmodulin modulation. Proc. Natl. Acad. Sci. USA 91, I I 757-I I 76 I.

Chen, T.-Y., Peng, V. W., Dhallan, R. S., Ahamed, B., Reed, R. R. \& Yau, K.-W. (1993). A new subunit of the cyclic nucleotide-gated cation channel in retinal rods. Nature (London) 362, 764-767.

Chen, T.-Y. \& Yau, K.-W. (r 994). Direct modulation by $\mathrm{Ca}^{2+}$-calmodulin of cyclic nucleotide-activated channel of rat olfactory receptor neurons. Nature (London) $\mathbf{3 6 8}$, $545^{-548 .}$

Colquhoun, D. (i 973). The relation between classical and cooperative models for drug action. In Drug Receptors (ed. H. P. Rang), pp. I49-182. New York: MacMillan.

del Castillo, J. \& Katz, B. ( I957). Interaction at end-plate receptors between different choline derivatives. Proc. R. Soc. London Ser. B r46, 369-38г.

Dostmann, W. \& Taylor, S. (i 99i). Identifying the molecular switches that determine $\left(\mathrm{R}_{\mathrm{p}}\right)$-cAMPs functions as an antagonist in the activation of cAMP-dependent protein kinase I. Biochemistry 30, 87 г $0-87$ I 6.

Fesenko, E. E., Kolesnikov, S. S. \& Lyubarsky, A. L. (1985). Induction by cyclic GMP of cationic conductance in plasma membrane of retinal rod outer segment. Nature (London) 3I3, 3 I0-3 I 3.

Finn, J. T., Grunwald, M. E. \& Yau, K.-W. (i996). Cyclic nucleotide-gated ion channels: an extended family with diverse functions. Annu. Rev. Physiol. 58, 395-426.

Galzi, J.-L. \& Changeux, J.-P. (i994). Neurotransmitter-gated ion channels as unconventional allosteric proteins. Curr. Opin. Struc. Biol. 4, 554-565.

Galzi, J.-L., Edelstein, S. \& Changeux, J.-P. (i 996). The multiple phenotypes of allosteric receptor mutants. Proc. Natl. Acad. Sci. USA 93, I853-1858.

Gordon, S., Brautigan, D. \& Zimmerman, A. (i 992). Protein phosphatases modulate the apparent agonist affinity of the light-regulated ion channel in retinal rods. Neuron 9, 739-748.

Gordon, S., Downing-Park, J., Tam, B. \& Zimmerman, A. (i $995 a$ ). Diacylglycerol analogs inhibit the rod cGMP-gated channel by a phosphorylation-independent mechanism. Biophys. F. 69, 409-4I7.

Gordon, S., Downing-Park, J. \& Zimmerman, A. (i995b). Modulation of the cGMPgated ion channel in frog rods by calmodulin and an endogenous inhibitory factor. $\mathcal{F}$. Physiol. 486, 533-546.

Gordon, S., Oakley, J., Varnum, M. \& Zagotta, W. (i 996). Altered ligand specificity by protonation in the ligand binding domain of cyclic nucleotide-gated channels. Biochemistry 35, 3994-400 I.

Gordon, S. \& Zagotta, W. (I995 $a$ ). A histidine residue associated with the gate of the cyclic nucleotide-activated channels in rod photoreceptors. Neuron I4, I77-1 83 .

Gordon, S. \& ZaGotTa, W. (I995 b). Localization of regions affecting an allosteric transition in cyclic nucleotide-activated channels. Neuron I4, 857-864.

Gordon, S. \& ZaGotTa, W. (I995c). Subunit interactions in coordination of $\mathrm{Ni}^{2+}$ in cyclic nucleotide-gated channels. Proc. Natl. Acad. Sci. USA 92, г0222-10226.

Goulding, E., Tibbs, G. \& Siegelbaum, S. (i 994). Molecular mechanism of cyclic nucleotide-gated channel activation. Nature (London) 372, 369-374.

Hammes, G. G. \& Wu, C.-W. (r97I). Regulation of enzyme activity. Science r72, I 205-I 2 II .

Haynes, L. \& Yau, K.-W. (I985). Cyclic GMP-sensitive conductance in outer segment membrane of catfish cones. Nature (London) 3I 7, 6I-64.

Heyduk, E., Heyduk, T. \& Lee, J. ( I 992). Intersubunit communications in E. coli cyclic 
I92 Fun Li et al.

AMP receptor protein: studies of the ligand binding domain. Biochemistry $\mathbf{3} \mathbf{r}$, $3682-3688$.

Hsu, Y.-T. \& Molday, R. S. (I993). Modulation of the cGMP-gated channel of rod photoreceptor cells by calmodulin. Nature (London) 36r, 76-79.

Ildefonse, M. \& Bennett, N. (i99i). Single-channel study of the cGMP-dependent conductance of retinal rods from incorporation of native vesicles into planar lipid bilayers. F. Mem. Biol. 123, I33-147.

JACKSON, M. (I993). The energetics of activation of membrane receptors. In Thermodynamics of Membrane Receptors and Channels (ed. M. Jackson), pp. 227-248. Boca Raton, FL: CRC Press.

Karlin, A. ( I 967). On the application of 'a plausible model' of allosteric proteins to the receptor for acetylcholine. F. Theor. Biol. 16, 306-320.

Karpen, J. \& Brown, R. (I996). Covalent activation of retinal rod cGMP-gated channels reveals a functional heterogeneity in the ligand binding sites. F. Gen. Physiol. Iо7, I69-i 8 I.

Karpen, J. W., Brown, R. L., Stryer, L. \& Baylor, D. A. (i993). Interactions between divalent cations and the gating machinery of cyclic GMP-activated channels in salamander retinal rods. F. Gen. Physiol. ror, I-25.

Körschen, H., Illing, M., Seifert, R., Sesti, F., Williams, A., Gotzes, S., Colville, C., Müller, F., Dosé, A., Godde, M., Molday, L., Kaupp, U. B. \& Molday, R. (I995). A $240 \mathrm{kDa}$ protein represents the complete $\beta$ subunit of the cyclic nucleotidegated channel from rod photoreceptor. Neuron 15, 627-636.

Koshland, D. E., Nemethy, G. \& Filmer, D. (i 966). Comparison of experimental binding data and theoretical models in proteins containing subunits. Biochemistry $\mathbf{5}$, $365-385$.

Kramer, R. \& Tibbs, G. (i 996 ). Antagonists of cyclic nucleotide-gated channels and molecular mapping of their site of action. F. Neurosci. 16, I 285-1 293.

Kumar, V. D. \& Weber, I. T. (r992). Molecular model of the cyclic GMP-binding domain of the cyclic GMP-gated ion channel. Biochemistry 31, 4643-4649.

Leff, P. ( I 995). The two-state model of receptor activation. Trends Pharmacol. Sci. r6, 89-97.

Liman, E. R. \& Buck, L. B. (i 994). A second subunit of the olfactory cyclic nucleotidegated channel confers high sensitivity to cAMP. Neuron 13, 6 I I-62 I.

Liu, M., Chen, T.-Y., Ahamed, B., Li, J. \& Yau, K.-W. (i 994). Calcium-calmodulin modulation of the olfactory cyclic nucleotide-gated cation channel. Science 266, I $348-$ I 354 .

Matthews, G. \& Watanabe, S.-I. (i 988). Activation of single channels from toad retinal rod inner segments by cyclic GMP: concentration dependence. F. Physiol. 403, $389-405$.

Molday, R. S. (1996). Calmodulin regulation of cyclic-nucleotide-gated channels. Curr. Opin. Neurobiol. 6, 445-452.

Monod, J., Wyman, J. \& Changeux, J.-P. (i965). On the nature of allosteric transitions: a plausible model. F. Mol. Biol. 12, 88-I I 8.

Picones, A. \& Korenbrot, J. (i 995$)$. Spontaneous, ligand-independent activity of the cGMP-gated ion channels in cone photoreceptors of fish. $\mathcal{F}$. Physiol. 585, 699-7I 4.

Ross, E. M. (I996). Pharmacodynamics: Mechanism of drug action and relationship between drug concentration and effect. In Goodman \& Gilman's The Pharmacological Basis of Therapeutics (ed. J. G. Hardman, L. E. Limbird, P. B. Molinoff, R. W. Ruddon and A. G. Gilman), pp. 29-42. McGraw-Hill. 
Sanfilippo, C. \& Menini, A. (i 993). Cytosolic acidification enhances the activation by cAMP of the channel in retinal rod outer segments. Biophys. $\mathcal{F} . \mathbf{6 4}$, Ar 7 .

Scholübbers, H.-G., Knippenberg, P. V., Baraniak, J., Stec, W., Morr, M. \& JASTORFF, B. (1984). Investigations on stimulation of lac transcription in vivo in Escherichia coli by cAMP analogues. Eur. F. Biochem. 138, го I-Iо9.

Scott, S.-P., Harrison, R., Weber, I. \& Tanaka, J. (i996). Predicted ligand interactions for $3^{\prime}, 5^{\prime}$-cyclic nucleotide gated channel binding sites: comparison of retina and olfactory binding site models. Protein Eng. 9, 333-344.

Serre, V., Ildefonse, M. \& Bennett, N. (i 995). Effects of cysteine modification on the activity of the cGMP-gated channel from retinal rods. F. Mem. Biol. I46, I45-I62.

Smith, G. \& Olsen, R. (i995). Functional domains of GABA $_{\mathrm{A}}$ receptors. Trends Pharmacol. Sci. 16, г62-168.

Stephenson, R. P. (1956). A modification of receptor theory. Br. F. Pharmacol. I 1 , 379-393.

TAYlor, W. R. \& Baylor, D. A. (I995). Conductance and kinetics of single cGMPactivated channels in salamander rod outer segments. $\mathcal{F}$. Physiol. 483, 567-582.

Tibbs, G., Goulding, E., Leypold, B., Liu, D. \& Siegelbaum, S. (i 996). Spontaneous opening of cyclic nucleotide-gated channels supports an allosteric model of activation. Biophys. F. 7o, A 137 .

Varnum, M., Black, K. \& Zagotta, W. (i995). Molecular mechanism for ligand discrimination of cyclic nucleotide-gated channels. Neuron I5, 6r9-625.

Varnum, M. D. \& Zagotta, W. N. (i996). Subunit interactions in the activation of cyclic nucleotide-gated ion channels. Biophys. F. 7o, 2667-2679.

Weber, I. T. \& Steitz, T. A. ( I 987). Structure of a complex of catabolite gene activator protein and cyclic AMP refined at 2.5 А resolution. F. Mol. Biol. 198, 3 I I-326.

Wyman, J. \& Gill, S. J. (1990). Binding and Linkage. Mill Valley, CA: University Science Books.

Zagotta, W. N. \& Siegelbaum, S. A. (i996). Structure and function of cyclic nucleotide-gated chanels. Annu. Rev. Neurosci. 19, 235-263.

Zhang, Y., Chen, J. \& Auerbach, A. (i995). Activation of recombinant mouse acetylcholine receptors by acetylcholine, carbamylcholine and tetramethylammonium. F. Physiol. 486, i 89-206.

Zimmerman, A. L. \& Baylor, D. A. (i 986). Cyclic GMP-sensitive conductance of retinal rods consists of aqueous pores. Nature (London) 321, 70-72. 